\title{
A novel media properties-based material removal rate model for magnetic field-assisted finishing
}

\author{
Chun Wai Kuma,b, Takashi Sato ${ }^{\mathrm{b}}$, Jiang Guo ${ }^{\mathrm{c}, \mathrm{d},{ }^{*}, \text { Kui Liud }}{ }^{\mathrm{d}}$, David Butler \\ a School of Mechanical and Aerospace Engineering, Nanyang Technological University, 50 \\ Nanyang Avenue, Singapore 639798 \\ ${ }^{b}$ Advanced Remanufacturing \& Technology Centre, 3 CleanTech Loop, \#01/01, CleanTech \\ Two, Singapore 637143 \\ cKey Laboratory for Precision and Non-traditional Machining Technology of Ministry of \\ Education, Dalian University of Technology, Dalian 116024, China \\ dSingapore Institute of Manufacturing Technology, 73 Nanyang Drive, Singapore 637662 \\ ${ }^{e}$ Design, Manufacture \& Engineering Management, The University of Strathclyde, Glasgow \\ G1 1XJ, United Kingdom
}

Email: guojiang3302@hotmail.com;

\section{Abstract}

Magnetic field assisted finishing (MFAF) is a category of non-conventional finishing processes that use magnetic field to manipulate finishing media typically consisting of magnetic particles and non-magnetic abrasives suspended in a carrier fluid. In order to better control the process, an improved understanding of the actual removal process is needed. This paper will introduce a new material removal rate model for magnetic fieldassisted finishing (MFAF) that will aim do so. The model considers the complexity of finishing media used in MFAF processes, where two different types of particles are presented and interact with each other. The proposed material removal rate expression is based on contact mechanics and is a function of the number of active magnetic particles, number of active abrasives, force per magnetic particle, and force per abrasive. Expressions for particle numbers have been developed by considering an ideal facecentred cubic configuration for the magnetic particle network, while expressions for forces have been developed based on a proposed framework for the particle interactions. The model has been verified experimentally for a double-magnet MFAF process by varying the abrasive size and abrasive concentration. When the abrasive size was increased from $0.6 \mu \mathrm{m}$ to $15 \mu \mathrm{m}$, the material removal rate decreased which is consistent 
with the theoretical trend given by the model. Then, when abrasive concentration, given by the abrasives-to-carbonyl-iron volumetric ratio, was increased from 0 to 0.768 , the material removal rate initially increased and then reached a maximum when the volume ratio is 0.259 before decreasing with further increase of the volume ratio. This is also in agreement with the theoretical trend given by the model.

Keywords: Magnetic field-assisted finishing; Abrasives; Material removal rate; Process modelling

\section{Nomenclatures}

a

$a_{\mathrm{Cl}} \quad$ Areal ratio of carbonyl iron particles on the $\mathrm{Cl}$ plane, $\Pi_{\mathrm{cl}}$

$A_{\text {abr }}$

$A_{\text {contact }}$

$A_{p}$

$B$

$C_{p}$

$D$

$D_{\text {abr }}$

$D_{\text {abreqv }}$

$D_{\text {abr,max }}$

$D_{\mathrm{Cl}}$

E

F

$F_{\text {abr }}$

$F_{\mathrm{Cl}}$

$f_{\text {abr }}$

$f_{\mathrm{Cl}}$

$h$

$k_{\text {abr }}$

$k_{\mathrm{Cl}}$

Radius of circle of intersection between carbonyl iron and the abrasive plane, $\prod_{\mathrm{abr}}$

Area available for abrasives on the abrasive plane, $\prod_{a b r}$

Area of contact between finishing media and workpiece

Projected area of indentation

Magnetic flux density

Constant of proportionality in Preston's equation

Particle size

Abrasive size

Equivalent abrasive size, in extension for abrasive volume criterion

Maximum allowable abrasive size as given by abrasive size criterion

Carbonyl iron particle size

Young's modulus

Force per particle

Force per abrasive

Force per carbonyl iron particle

Force factor for abrasives

Force factor for carbonyl iron particles

Indentation depth of particle

Removal factor for abrasives

Removal factor for carbonyl iron particles 


$\begin{array}{ll}\Delta \mathrm{MRR}_{\mathrm{abr}} & \text { Material removal rate per abrasive } \\ \Delta \mathrm{MRR}_{\mathrm{Cl}} & \text { Material removal rate per carbonyl iron particle } \\ \mathrm{MRR} & \text { Material removal rate } \\ N & \text { Number of active particle } \\ N_{\mathrm{abr}} & \text { Number of active abrasives } \\ N_{\mathrm{abr}, \mathrm{max}} & \text { Number of active abrasives at abrasive saturation } \\ N_{\mathrm{Cl}} & \text { Number of active carbonyl iron particles } \\ P & \text { Pressure on workpiece in Preston's equation } \\ \prod_{\mathrm{abr}} & \text { Abrasive plane, where calculation of } N_{\mathrm{abr}} \text { is considered } \\ \prod_{\mathrm{cl}} & \text { Carbonyl iron particle plane, where calculation of } N_{\mathrm{Cl}} \text { is considered } \\ V_{\mathrm{abr}} / V_{\mathrm{FCC}} & \text { Volumetric ratio of abrasives in the FCC unit cube } \\ V_{\mathrm{abr}} / V_{\mathrm{Cl}} & \text { Volumetric ratio of abrasives to carbonyl iron } \\ V_{\mathrm{abr}, \mathrm{max}} / V_{\mathrm{Cl}} & \text { Maximum allowable volumetric ratio of abrasives in the } \mathrm{FCC} \text { unit cube } \\ V_{\mathrm{Cl}} / V_{\mathrm{FCC}} & \text { Volumetric ratio of carbonyl iron in the FCC unit cube } \\ V_{r} & \text { Relative velocity between workpiece and tool }\end{array}$

\section{Introduction}

Magnetic field-assisted finishing (MFAF) is a category of non-conventional finishing processes that utilize magnetic fields to manipulate the properties of the finishing media typically consisting of magnetic particles such as carbonyl iron, non-magnetic abrasives, carrier fluid such as water, and chemical additives. MFAF is usually employed as the final step in manufacturing processes for the purpose of surface roughness reduction, removal of sub-surface damage layer or for form error correction. Under the influence of magnetic field, magnetic particles in the finishing media form chains, resulting in significant increase of the viscosity of finishing media, usually by several orders of magnitude. This stiffened finishing media can then function as a conformable polishing lap. When this stiffened finishing media is brought into contact with the workpiece surface and a relative motion is introduced, material removal from the workpiece surface occurs. The unique behavior of the finishing media has resulted in the development of many different finishing techniques that manipulate the finishing media in different ways to achieve a wide array of goals. The most well-known is arguably the Magnetorheological Finishing (MRF) process, which is commercially available for superfinishing of high-value 
optics components [1]. Other MFAF processes include the magnetic abrasive finishing (MAF) process [2], an apparatus for finishing of ground glass edge [3], a double-magnet configuration for high material removal rate $[4,5]$ that can also be mounted on a robotic arm [6], an apparatus for finishing of internal surface of tubes [7], an apparatus for finishing of micropore X-ray focusing mirrors [8], and a ball-end tool [9].

One of the key research issues for MFAF processes is the prediction of material removal rate based on a given set of input process parameters. The ability to predict the material removal rate is advantageous for reducing or eliminating trial-and-error when setting up the process conditions for a new task. Additionally, it is also critical for the generation of tool path for process automation. However, a holistic knowledge of the underlying material removal mechanism does not currently exist [10], even though the relationships between material removal rate and process parameters have been quite well established for some processes [11].

One of the earliest material removal rate models for abrasive processes was proposed by Preston [12] to model the material removal rate for lapping of glass plates with a felt lap. According to Preston's equation:

$$
\mathrm{MRR}=C_{P} P v_{r}
$$

where MRR is the material material removal rate, $P$ is the polishing pressure, $v_{r}$ is the relative velocity and $C_{P}$ is a constant of proportionality that embodies other process factors such as workpiece material properties, abrasives material properties, tooling parameters, and chemistry. Preston's equation has been modified and extended by several newer models [13, 14], some of which are targeted at MFAF processes. Other novel models that consider macroscale factors such as rotation speed and magnetic field strength have also been proposed in the literature $[15,16]$. However, these models do not consider the mechanics and interactions of the two types of particles in MFAF finishing media. Contact mechanics is another common approach in modeling material removal rate, especially for chemical-mechanical polishing (CMP) [17, 18]. These models place more emphasis on the particle mechanics in the finishing media, but CMP media is composed only of a single particle type and therefore does not reflect the mechanics of MFAF finishing media. Finishing forces have been considered for MFAF [19], although the multivariate regression approach generated results that are specific to the experimental setup reported. In summary, existing material removal rate models for 
abrasive processes do not represent the complexity of the finishing media used in MFAF processes.

Within this work, a material removal rate model based on contact mechanics that considers the complexity of the finishing media in the MFAF process is proposed and developed. A new framework to quantify the interactions between the two types of particles in the finishing media is also proposed. The model is then validated experimentally for the double-magnet MFAF process, which has been introduced elsewhere [4].

\section{Material removal rate model}

\subsection{Assumptions}

Firstly, the carbonyl iron particles and abrasives are assumed to be spherical in shape with a uniform size distribution. Spherical carbonyl iron particles are commonly available and alumina abrasives can be approximated to be spherical, as shown in scanning electron micrographs in Figure 1. Assumption of uniform size distribution is necessary to simplify the model formulation. Secondly, the carbonyl iron particles are assumed to pack in a face-centred cubic (FCC) configuration under the influence of a magnetic field. This mirrors the behavior of carbonyl iron particles in a magnetic field, where they are strongly attracted to each other, resulting in a close-packed configuration, and FCC has the highest packing factor of 0.74 [20]. Lastly, the abrasives are assumed to occupy the interstitial spaces in the carbonyl iron network without compromising the FCC configuration of the network. For the last assumption to be valid, the abrasive size must adhere to the abrasive size criterion, which is a condition typical to magnetic finishing processes $[4,5,14]$ :

$$
D_{a b r} \leq(\sqrt{2}-1) \cdot D_{C l}
$$

where $D_{\mathrm{abr}}$ is the diameter of abrasives and $D_{\mathrm{Cl}}$ is the diameter of carbonyl iron particles. Equation 2 is obtained by considering the unit cube shown in Figure 2. Consequently, the maximum allowable abrasive size, $D_{\mathrm{abr}, \max }$, is given by $(\sqrt{ } 2-1) D_{\mathrm{Cl}}$.

A maximum allowable abrasive concentration also exists because of finite interstitial spaces in the carbonyl iron network. Within this work, abrasive concentration is quantified by the abrasive-to-carbonyl-iron volumetric ratio, $V_{\mathrm{abr}} / V_{\mathrm{Cl}}$. In the FCC unit cube, the 
volume fraction of carbonyl iron particles, $V_{\mathrm{Cl}} / V_{\mathrm{FCC}}$, is 0.74 . The volume fraction of interstitial spaces is therefore 0.26 . To obtain the theoretical maximum volume fraction of abrasives, $V_{\mathrm{abr}, \mathrm{max}} / V_{\mathrm{FCC}}$, the maximum packing factor of 0.74 is applied to the interstitial spaces, resulting in a value of 0.192 for $V_{\text {abr,max }} / V_{\mathrm{FCC}}$. Consequently, the abrasive volume criterion is given by:

$$
V_{\mathrm{abr}} / V_{\mathrm{Cl}} \leq 0.259
$$

where the value of 0.259 is the ratio of $V_{\mathrm{abr}, \max } / V_{\mathrm{Cl}}$.

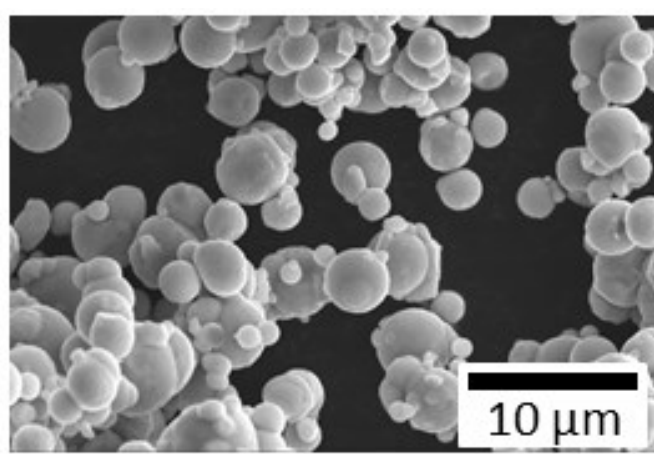

(a)

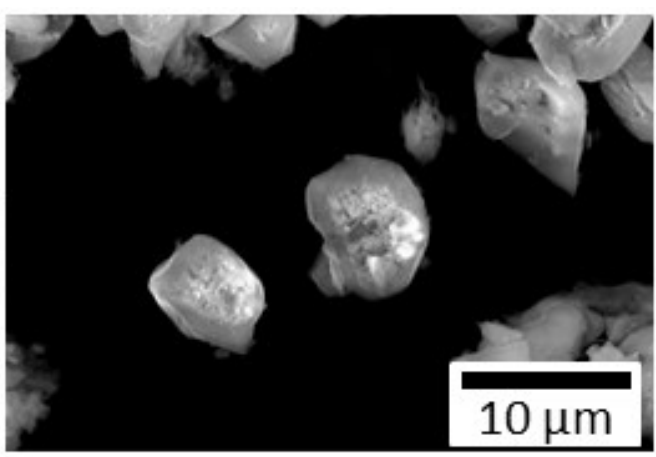

(b)

Figure 1: Scanning electron micrographs of (a) carbonyl iron particles, and (b) alumina abrasives.

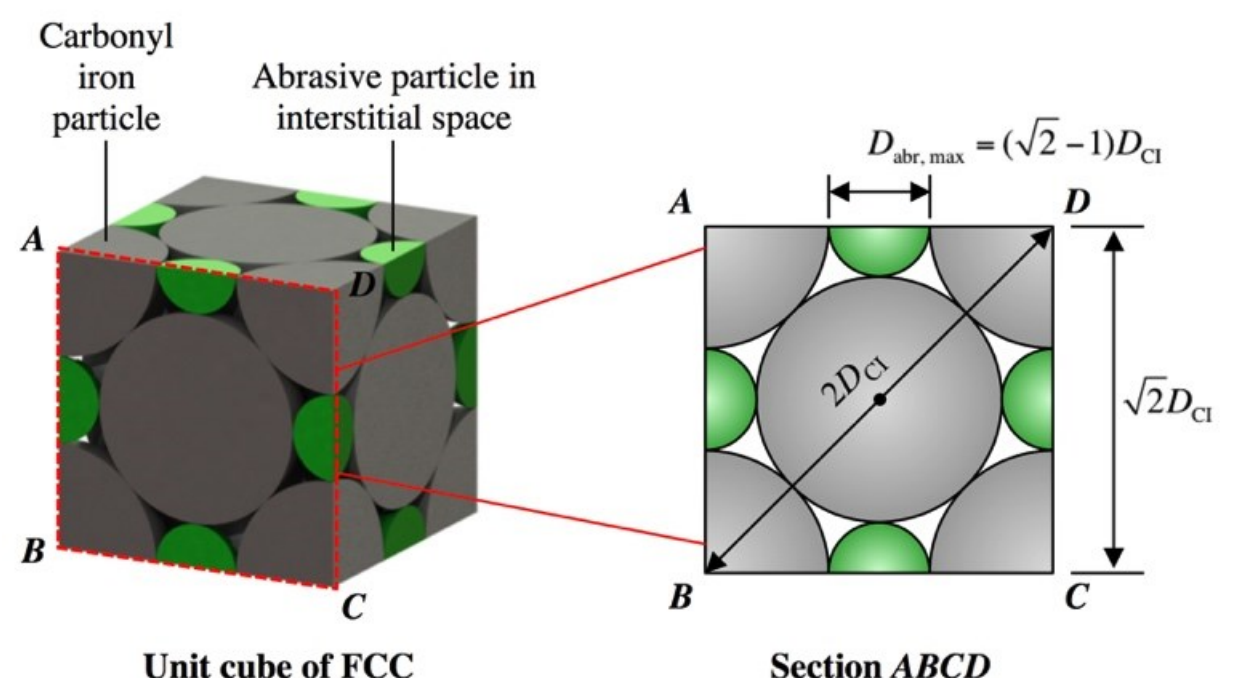

Figure 2: Schematic diagram of FCC unit cube of the carbonyl iron network. 


\subsection{General material removal rate expression}

Total material removal rate is considered to be the sum of two components, where one is attributed to carbonyl iron particles and the other to abrasives. Each component is the product of the number of active particles and volume removed per particle. A particle is considered as active if it is in contact with the workpiece surface during finishing. In addition, the constants $k_{\mathrm{Cl}}$ and $k_{\mathrm{abr}}$ are introduced to account for the different material removal efficiency of the two types of particles, where it is expected that $k_{\mathrm{abr}} \gg k_{\mathrm{Cl}}$. The total material removal rate is then given by:

$$
\mathrm{MRR}=k_{\mathrm{Cl}} \cdot N_{\mathrm{Cl}} \cdot \Delta \mathrm{MRR}_{\mathrm{Cl}}+k_{\mathrm{abr}} \cdot N_{\mathrm{abr}} \cdot \Delta \mathrm{MRR}_{\mathrm{abr}}
$$

where $N_{\mathrm{Cl}}$ and $N_{\mathrm{abr}}$ are the numbers of active carbonyl iron particles and abrasives respectively, and $\Delta M R R_{C l}$ and $\Delta M R R_{a b r}$ are the material removal rates per carbonyl iron particle and per abrasive respectively.

To calculate $\Delta M R R_{C l}$ and $\Delta M R R_{a b r}$, Hertz's equation for shallow spherical indentation of a half space [21] is considered. Referring to Figure 3, Hertz's equation gives the depth of particle indentation $h$ as:

$$
h=\left(\frac{3 F}{8 E}\right)^{2 / 3} \cdot \frac{1}{D^{1 / 3}}
$$

where $F$ is the force per particle, $E$ is the Young's modulus and $D$ is the particle diameter. By considering the material removal rate per particle as $A_{p} v_{r}$ where $A_{p}$ is the projected area of indentation and $v_{r}$ the relative velocity, and the approximation $A_{p}^{2}=h^{3} D$, Equation 4 can be rewritten as:

$$
\mathrm{MRR}=k_{\mathrm{Cl}} \cdot N_{\mathrm{Cl}} \cdot \frac{3 F_{\mathrm{Cl}}}{8 E} \cdot v_{r}+k_{\mathrm{abr}} \cdot N_{\mathrm{abr}} \cdot \frac{3 F_{\mathrm{abr}}}{8 E} \cdot v_{r}
$$

where $F_{\mathrm{Cl}}$ and $F_{\mathrm{abr}}$ are the forces per carbonyl iron particle and per abrasive respectively. Equation 6 is generalized for all MFAF processes with finishing media consisting of two types of particles. In the following sections, the expressions for $N_{\mathrm{Cl}}, N_{\mathrm{abr}}, F_{\mathrm{Cl}}$ and $F_{\mathrm{abr}}$ are developed. 


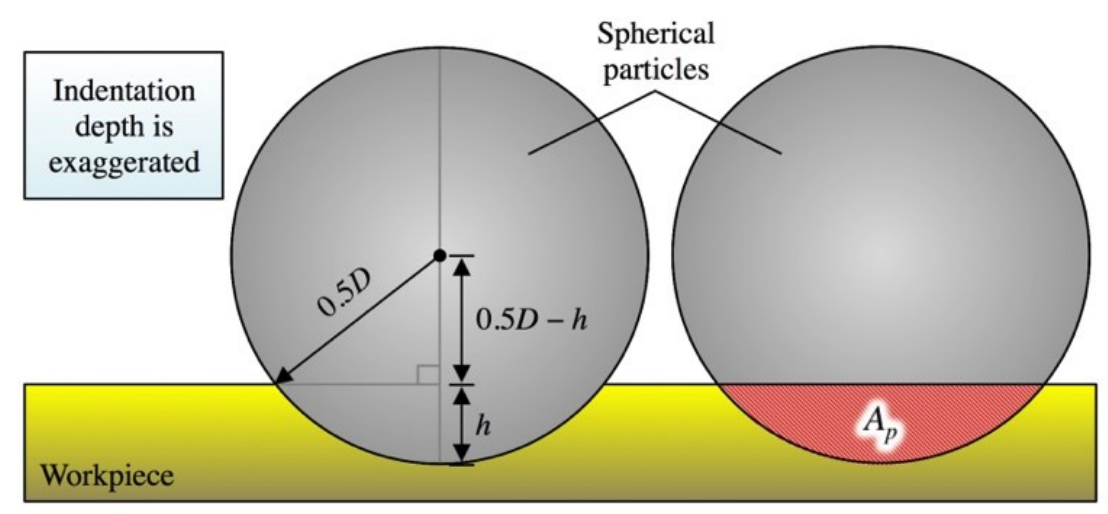

Figure 3: Schematic diagram of the depth of indentation and projected area of indentation.

\subsection{Number of active particles}

For the model, a particle is considered as active if it is in contact with the workpiece surface. For MFAF processes, the number of active carbonyl iron particles, $N_{\mathrm{Cl}}$, and number of active abrasives, $N_{\mathrm{abr}}$, must both be considered. To that end, the planes $\prod_{\mathrm{c}}$ and $\prod_{a b r}$ are considered, as shown in Figure $4 . \prod_{c ı}$ and $\prod_{\text {abr }}$ are parallel to the workpiece surface, with offsets of $0.5 D_{\mathrm{Cl}}$ and $0.5 D_{\mathrm{abr}}$ respectively.

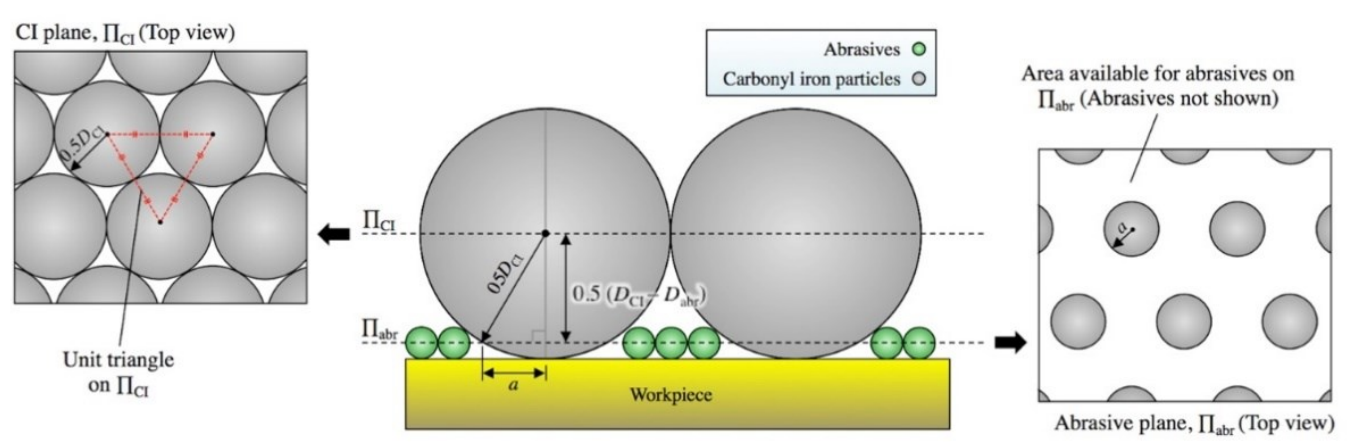

Figure 4: Schematic diagram of $\prod_{c}$ and $\prod_{a b r}$, and the unit triangle on $\prod_{c}$

$N_{\mathrm{cl}}$ is calculated on $\prod_{\mathrm{cl}}$, while $N_{\mathrm{abr}}$ is calculated on $\prod_{\mathrm{abr}}$. On $\prod_{\mathrm{cl}}$, a unit equilateral triangle, as shown in Figure 4, is considered. In this unit triangle, the ratio of area occupied by carbonyl iron particles, $a_{\mathrm{cl}}$, is calculated to be $\pi /(2 \sqrt{3})$ or 0.907 . Over the whole area of contact between finishing media and workpiece, $A_{\text {contact, }}$ the area occupied by carbonyl iron particles is therefore $a_{\mathrm{cl}} A_{\text {contact. }} N_{\mathrm{Cl}}$ can then be obtained by dividing this by the projected area of one carbonyl iron particle. Consequently, $N_{C I}$ is given by: 


$$
N_{\mathrm{Cl}}=\frac{2}{\sqrt{3}} \cdot \frac{A_{\text {contact }}}{D_{\mathrm{Cl}}{ }^{2}}
$$

Next, the plane $\prod_{\mathrm{abr}}$ is considered. Some area on the plane is occupied by the existing carbonyl iron particles. The theoretical maximum area available for abrasives on $\prod_{a b r}$, $A_{\mathrm{abr}}$, is the remaining area with the ratio $\pi /(2 \sqrt{3})$ applied. As a result, $A_{\mathrm{abr}}$ is then given by:

$$
A_{\mathrm{abr}}=\frac{\pi}{2 \sqrt{3}} \cdot\left(A_{\text {contact }}-N_{\mathrm{Cl}} \pi a^{2}\right)
$$

where $a$ is radius of the circle of intersection between a carbonyl iron particle and $\prod_{a b r}$, and is given by $0.25 D_{\text {abr }}\left(2 D_{\mathrm{Cl}}-D_{\mathrm{abr}}\right)$. With the same method used for $N_{\mathrm{Cl}}$, the maximum number of abrasives on $\prod_{\mathrm{abr}}, N_{\mathrm{abr}, \max }$ is then given by:

$$
N_{\mathrm{abr}, \max }=\frac{2}{\sqrt{3}} \cdot \frac{A_{\mathrm{contact}}}{D_{\mathrm{abr}}{ }^{2}} \cdot\left(1-\frac{\pi}{2 \sqrt{3}} \cdot \frac{D_{\mathrm{abr}}}{D_{\mathrm{Cl}}} \cdot \frac{2 D_{\mathrm{Cl}}-D_{\mathrm{abr}}}{D_{\mathrm{Cl}}}\right)
$$

$N_{\text {abr,max }}$ coincides with the maximum abrasive volume given by the abrasive volume criterion in Equation 3. To obtain $N_{\text {abr }}$, the following relationship is considered between number of active abrasives and volume of abrasives:

$$
\frac{N_{\mathrm{abr}}}{N_{\mathrm{abr}, \max }}=\frac{V_{\mathrm{abr}} / V_{\mathrm{CI}}}{0.259}
$$

As a result, the expression of $N_{\text {abr }}$ is given by:

$$
N_{\mathrm{abr}}=\frac{2}{\sqrt{3}} \cdot \frac{A_{\mathrm{contact}}}{D_{\mathrm{abr}}{ }^{2}} \cdot\left(1-\frac{\pi}{2 \sqrt{3}} \cdot \frac{D_{\mathrm{abr}}}{D_{\mathrm{Cl}}} \cdot \frac{2 D_{\mathrm{Cl}}-D_{\mathrm{abr}}}{D_{\mathrm{Cl}}}\right) \cdot \frac{V_{\mathrm{abr}} / V_{\mathrm{CI}}}{0.259}
$$

\subsection{Force per particle}

Force per particle for MFAF processes is unique because it is attributed to the contact forces of the neighbouring particles. For $F_{\mathrm{Cl}}$ and $F_{\mathrm{abr}}$, it is proposed that they are each the product of two factors. The first factor is related to the properties of the particle itself, while the second factor is related to the composition and properties of the neighbouring particles. This can be written generally as:

$$
F_{\mathrm{Cl}} \text { or } F_{\mathrm{abr}}=(\text { particle property factor }) \times(\text { neighbouring particles factor })
$$


For $F_{\mathrm{Cl}}$, the particle property factor is expressed as $B D_{\mathrm{Cl}}{ }^{3}$, where $B$ is the magnetic flux density at the centre of the particle. The term $D_{\mathrm{Cl}}{ }^{3}$ is attributed to the magnetic force exerted on the particle, which is proportional to the volume of the particle.

The formulation for the neighbouring particles factor is considerably more challenging, given that the configuration and composition of the neighbouring particles are stochastic in nature. In this preliminary work, an expression is therefore obtained based on several simplifying considerations. Firstly, the neighbouring particles factor is proposed to be the sum of two terms, one attributed to the neighbouring carbonyl iron particles, and another to the neighbouring abrasives. Secondly, each term is assigned a weightage to reflect the composition of the two types of neighbouring particles. Specifically, the weightage is based on the ratio of projected area of the particle type to the total projected area of all active particles. Projected area is selected as the basis of the ratio because contact force is proportional to the projected area of the particle. Effectively, the configuration of neighbouring particles is not considered. Thirdly, the constants $f_{\mathrm{Cl}}$ and $f_{\mathrm{abr}}$ are assigned to the two terms to account for difference in magnitude of the contact forces for the two types of particles. It is anticipated that $f_{\mathrm{Cl}} \gg f_{\mathrm{abr}}$ because carbonyl iron particles are strongly held by magnetic force, while abrasives are simply supported by neighbouring particles. Finally, an additional $B D_{\mathrm{Cl}}{ }^{3}$ is multiplied to the carbonyl iron particle term to reflect the dependence on magnetic flux density and particle volume. All these considered, the expression for $F_{\mathrm{Cl}}$ is then given by:

$$
F_{\mathrm{Cl}}=B D_{\mathrm{Cl}}{ }^{3} \cdot\left(f_{\mathrm{Cl}} \cdot B D_{\mathrm{Cl}}{ }^{3} \cdot \frac{N_{\mathrm{Cl}} D_{\mathrm{Cl}}{ }^{2}}{N_{\mathrm{Cl}} D_{\mathrm{Cl}}{ }^{2}+N_{\mathrm{abr}} D_{\mathrm{abr}}{ }^{2}}+f_{\mathrm{abr}} \cdot \frac{N_{\mathrm{abr}} D_{\mathrm{abr}}{ }^{2}}{N_{\mathrm{Cl}} D_{\mathrm{Cl}}{ }^{2}+N_{\mathrm{abr}} D_{\mathrm{abr}}{ }^{2}}\right)
$$

For $F_{\text {abr }}$, the particle property factor is expressed as $D_{\text {abr }}{ }^{2}$, as abrasives are not influenced by magnetic flux density and the dominant factor is therefore the contact force, which is proportional to the projected area of the particle itself. The neighbouring particles factor is the same as that derived for $F_{\mathrm{Cl}}$, since the active particles are considered as a bulk. $F_{\text {abr }}$ is therefore given by:

$$
F_{\mathrm{abr}}=D_{\mathrm{abr}}{ }^{2} \cdot\left(f_{\mathrm{Cl}} \cdot B D_{\mathrm{Cl}}{ }^{3} \cdot \frac{N_{\mathrm{Cl}} D_{\mathrm{Cl}}{ }^{2}}{N_{\mathrm{Cl}} D_{\mathrm{Cl}}{ }^{2}+N_{\mathrm{abr}} D_{\mathrm{abr}}{ }^{2}}+f_{\mathrm{abr}} \cdot \frac{N_{\mathrm{abr}} D_{\mathrm{abr}}{ }^{2}}{N_{\mathrm{Cl}} D_{\mathrm{Cl}}{ }^{2}+N_{\mathrm{abr}} D_{\mathrm{abr}}{ }^{2}}\right)
$$

With that, the expressions for $N_{\mathrm{Cl}}, N_{\mathrm{abr}}, F_{\mathrm{abr}}$ and $F_{\mathrm{Cl}}$ have all been developed. The derivation of the material removal rate model is thus complete. 


\subsection{Extension for abrasive size criterion}

The base model developed in the previous section requires the FCC carbonyl iron network to be unperturbed. When abrasive size exceeds the maximum allowable size given by the abrasive size criterion in Equation 2, the carbonyl iron network is compromised because additional space must be created to accommodate larger abrasives. The base model therefore becomes invalid. However, it is not inconceivable for finishing media to be composed of abrasives larger than the maximum allowable size. Therefore, the base model needs to be extended to consider such conditions.

To that end, an orderly increase in the distance between carbonyl iron particles in the carbonyl iron network is considered, resulting in an enlarged unit cube as shown in Figure 5. Consequently, the new distance between the centres of two adjacent carbonyl iron particles is now given by:

$$
\frac{1}{\sqrt{2}}\left(D_{\mathrm{Cl}}+D_{\mathrm{abr}}\right)
$$

This change in the distance between two adjacent carbonyl iron particles propagates to the unit triangle previously considered on the $\prod_{c ı}$ plane. This change in the unit triangle requires the expressions of $N_{\mathrm{Cl}}$ and $N_{\mathrm{abr}}$ to be modified accordingly. Following the same derivation done for the base model, the following expressions for $N_{\mathrm{Cl}}$ and $N_{\mathrm{abr}}$ are obtained:

$$
\begin{aligned}
& N_{\mathrm{Cl}}=\frac{4}{\sqrt{3}} \cdot \frac{A_{\text {contact }}}{\left(D_{\mathrm{Cl}}+D_{\mathrm{abr}}\right)^{2}} \\
& N_{\mathrm{abr}}=\frac{2}{\sqrt{3}} \cdot \frac{A_{\mathrm{contact}}}{D_{\mathrm{abr}}{ }^{2}} \cdot\left(1-\frac{\pi}{2 \sqrt{3}} \cdot \frac{2 D_{\mathrm{abr}}}{D_{\mathrm{Cl}}+D_{\mathrm{abr}}} \cdot \frac{2 D_{\mathrm{Cl}}-D_{\mathrm{abr}}}{D_{\mathrm{Cl}}+D_{\mathrm{abr}}}\right) \cdot \frac{V_{\mathrm{abr}} / V_{\mathrm{CI}}}{0.259}
\end{aligned}
$$

The above expressions are valid when the abrasive size criterion given in Equation 2 is not met. Expressions derived earlier for $F_{\mathrm{Cl}}$ and $F_{\mathrm{abr}}$ are not affected by the changes in the carbonyl iron network, since they consider the active particles in bulk and are therefore independent of the configuration of particles. Therefore, expressions derived earlier are still valid. However, note that the values of $F_{\mathrm{Cl}}$ and $F_{\mathrm{abr}}$ will change because both are functions of $N_{\mathrm{abr}}$ and $N_{\mathrm{Cl}}$ and their expressions have been modified. 


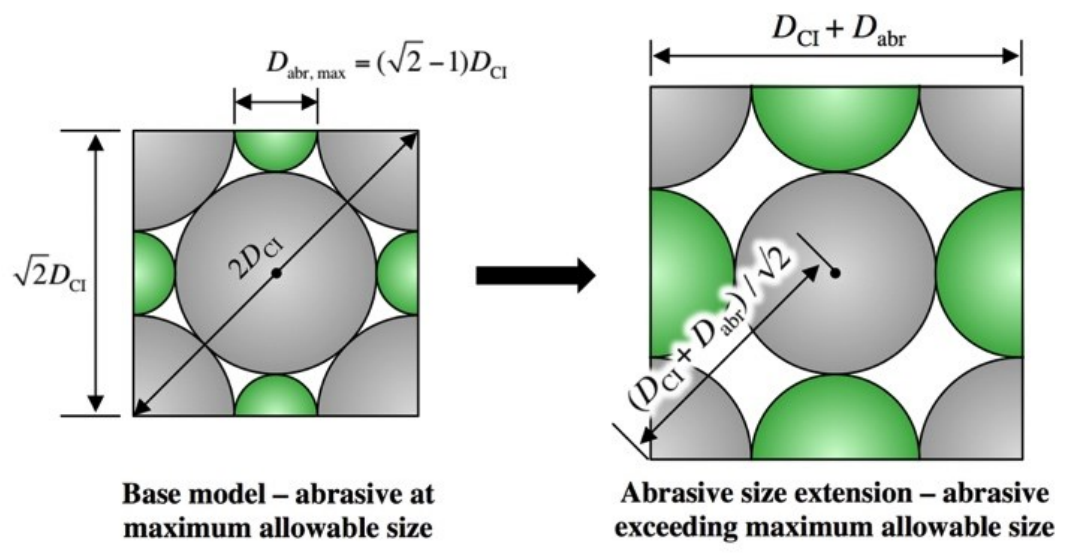

Figure 5: Schematic diagram of changes in FCC unit cube for the abrasive size extension to base model.

\subsection{Extension for abrasive volume criterion}

Similarly, an abrasive concentration exceeding the maximum allowable volume given by abrasive volume criterion in Equation 3 compromises the carbonyl iron network because additional space must be created to accommodate the extra abrasives. Therefore, the base model becomes invalid. However, it is not inconceivable for abrasive concentration to exceed the maximum allowable volume. To account for such conditions, an equivalent abrasive size is considered. The equivalent abrasive size, $D_{\text {abreqv, }}$ is obtained by considering the following relation:

$$
\frac{\pi D_{\mathrm{abr}, \mathrm{eqv}}{ }^{2}}{4}=\frac{\pi D_{\mathrm{abr}, \mathrm{max}}{ }^{2}}{4} \cdot \frac{V_{\mathrm{abr}}}{V_{\mathrm{abr}, \mathrm{max}}}
$$

which then results in:

$$
D_{\mathrm{abr}, \text { eqv }}=(\sqrt{2}-1) D_{\mathrm{Cl}} \cdot\left(\frac{V_{\mathrm{abr}}}{V_{\mathrm{abr}, \max }}\right)^{1 / 2}
$$

Since $D_{\text {abreqv }}$ is greater than $D_{\text {abr,max, }}$ the subsequent calculations are reduced to the case described in the previous section, where the abrasive size criterion is not met. Expressions developed for the extension for abrasive size criterion are therefore applicable, with $D_{\text {abreqv }}$ replacing the $D_{\text {abr }}$ terms when appropriate. The expressions for $N_{\mathrm{Cl}}$ and $N_{\mathrm{abr}}$ are eventually given by: 


$$
\begin{aligned}
& N_{\mathrm{Cl}}=\frac{4}{\sqrt{3}} \cdot \frac{A_{\text {contact }}}{\left(D_{\mathrm{Cl}}+D_{\mathrm{abr}, \text { eqv }}\right)^{2}} \\
& N_{\mathrm{abr}}=\frac{2}{\sqrt{3}} \cdot \frac{A_{\text {contact }}}{D_{\mathrm{abr}}{ }^{2}}\left(1-\frac{\pi}{2 \sqrt{3}} \cdot \frac{2 D_{\mathrm{abr}}}{D_{\mathrm{Cl}}+D_{\mathrm{abr}, \text { eqv }}} \cdot \frac{2 D_{\mathrm{Cl}}-D_{\mathrm{abr}}}{D_{\mathrm{Cl}}+D_{\text {abr,eqv }}}\right) \cdot \frac{V_{\mathrm{abr}} / V_{\mathrm{CI}}}{0.259}
\end{aligned}
$$

For $N_{\text {abr }}$, it is to be noted that not all $D_{\text {abr }}$ terms have been replaced by $D_{\text {abreqv. }}$ This is because the $\prod_{\text {abr }}$ plane is still considered to be of a distance of $D_{\text {abr }}$ from the workpiece surface, instead of $D_{\text {abreqv. }}$. As such, $D_{\text {abr }}$ terms attributed to the position of the $\prod_{\text {abr }}$ plane

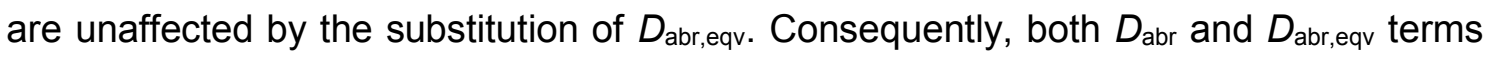
exist in the expression for $N_{\text {abr. }}$

The expressions for both $F_{\mathrm{Cl}}$ and $F_{\mathrm{abr}}$ are similarly unaffected by the use of $D_{\text {abreqv }}$, although it is to be noted that the values of $F_{\mathrm{Cl}}$ and $F_{\mathrm{abr}}$ will change because both are functions of $N_{\text {abr }}$ and $N_{\mathrm{Cl}}$.

In summary, the proposed model can be applied as a theoretical performance assessment of different mixing ratio of the ingredient in finishing media. The model can be applied in complement to material removal model that focuses on macroscale factors, such as rotation speed and magnetic field strength to provide greater insight into magnetic field-assisted finishing processes. In future, there is potential in expanding the model to consider other factors such as surface morphology, chemical properties and distribution of particle sizes.

\section{Experimental setup}

Experiments were conducted to validate the proposed model and its two extensions for an MFAF process that uses a double-magnet apparatus, which is shown in the photographs and schematic diagram in Figure 6 [4]. The polishing unit consists of two parallel shafts with neodymium ring magnets (Grade N35, $30 \mathrm{~mm}$ OD, $18.5 \mathrm{~mm}$ ID, 11 $\mathrm{mm}$ thickness) attached to each end, with an air gap of $1 \mathrm{~mm}$ maintained between the two magnets. One of the shafts is driven by a spindle motor and is connected to the other shaft by spur gears. As a result, the two magnets rotate in opposite directions. The whole unit is mounted on a desktop milling machine. Hence, the feed in the $x, y$, and $z$ axes as well as the spindle revolution speed are all numerically controlled. Finishing media consisting of carbonyl iron particles, alumina abrasives and water is then supplied to the magnets. Finishing media is prepared before each experimental run by manually mixing 
the three components. No surfactant or chemical additives are used due to the short polishing duration (5 minutes for each dose of finishing media). During polishing, a gap of $1 \mathrm{~mm}$ is maintained between the workpiece and the bottom surface of the two rotating magnets. This gap will be occupied by finishing media. In essence, material removal is achieved by the combination of force exerted by finishing media on the workpiece, and the relative movement between the finishing media and the workpiece. The doublemagnet configuration is advantageous firstly due to the high magnetic flux density in the polishing spot, and secondly due to the ability for the finishing media to be redressed in situ during polishing. Figure 7 illustrates the distribution of magnetic flux density and the magnetic flux lines given by magnetic field distribution analysis with the ANSYS software package. The simulated value of magnetic flux density at the polishing spot (centre position, $1 \mathrm{~mm}$ away from the magnets) is $0.637 \mathrm{~T}$. Measurement with a gaussmeter gave an averaged value within $0.05 \mathrm{~T}$. Both of these factors result in a relatively high material removal rate for this MFAF process. The principles of the process and its characteristics have been reported in detail elsewhere [4]. For experiments, polishing is done at a single spot without any tool feed for a duration of 5 minutes for each condition. 


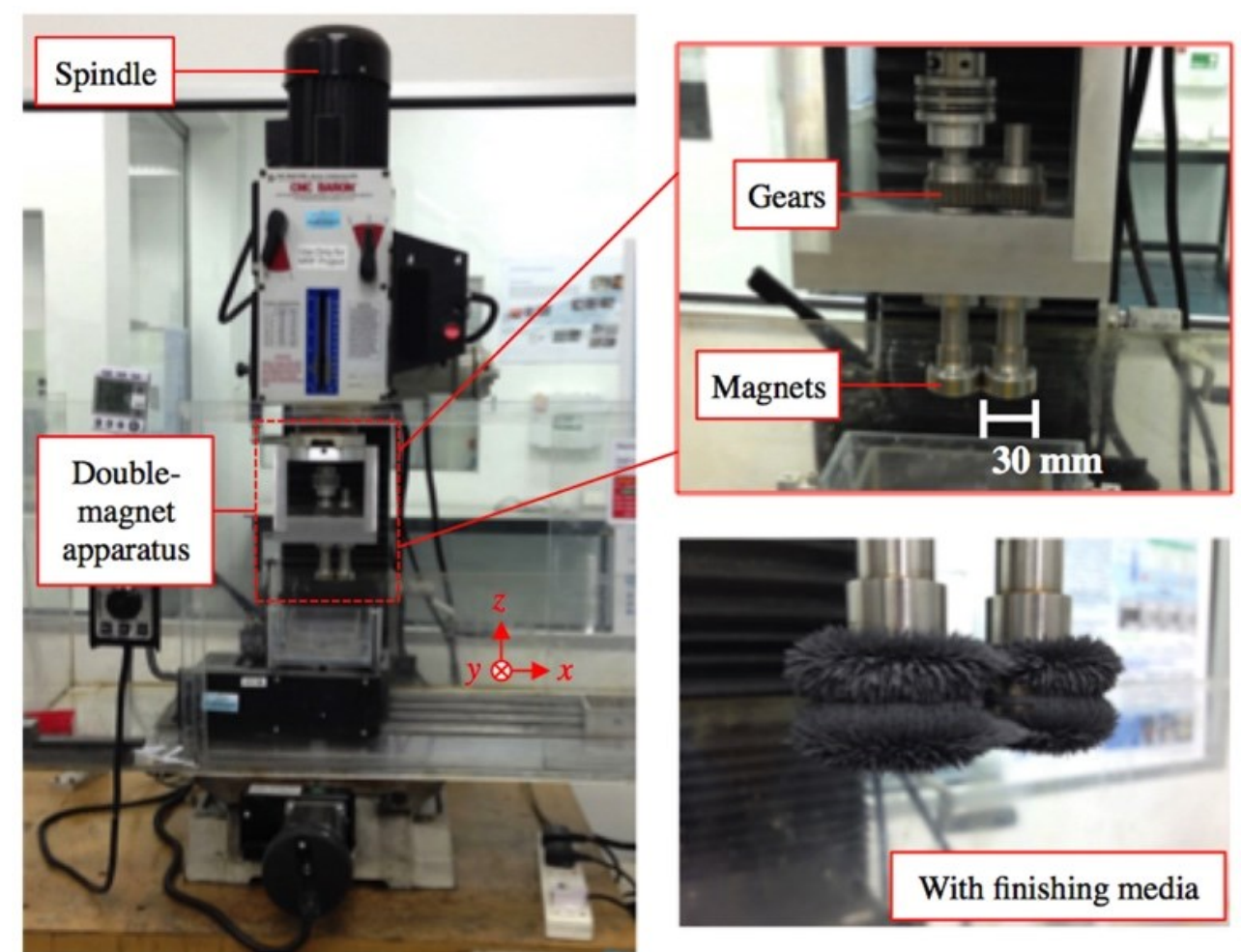

(a)

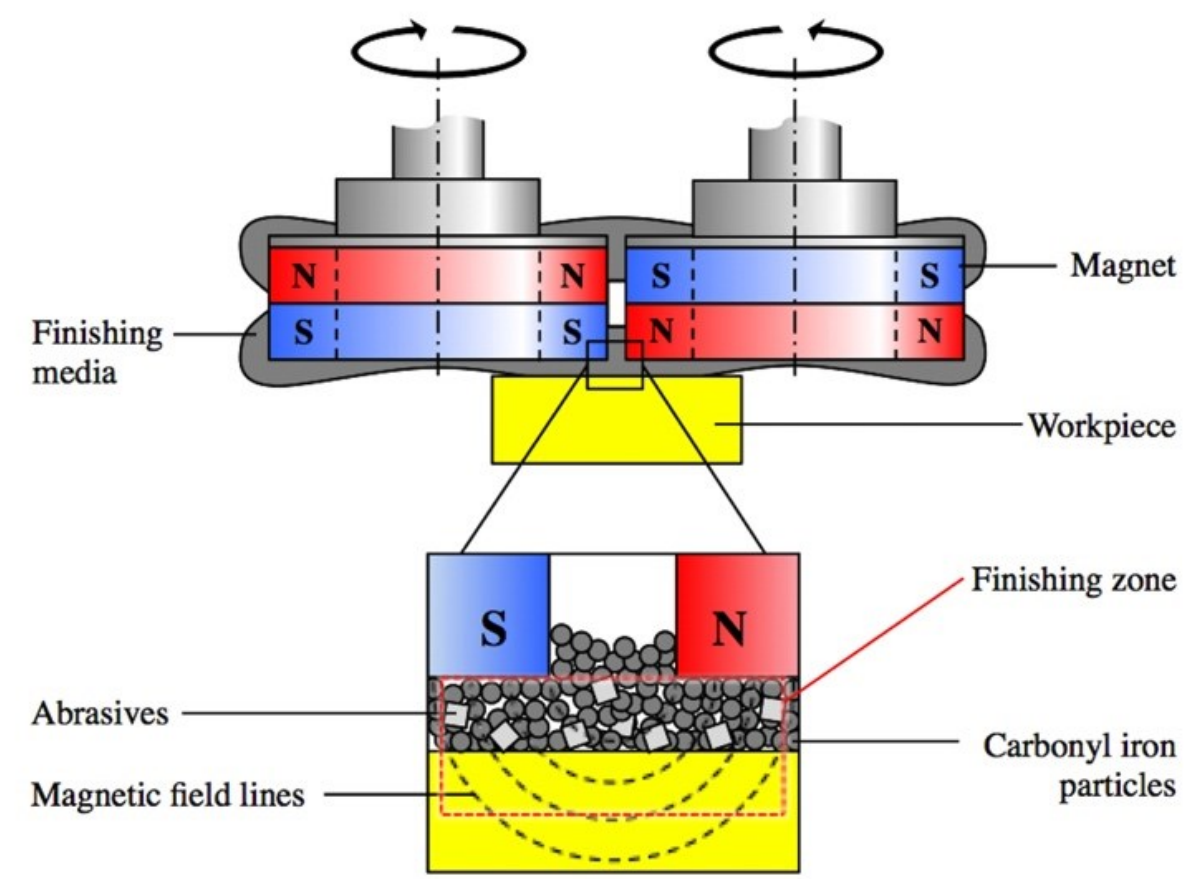

(b)

Figure 6: (a) Photographs and (b) schematic diagram of the double-magnet MFAF apparatus. 


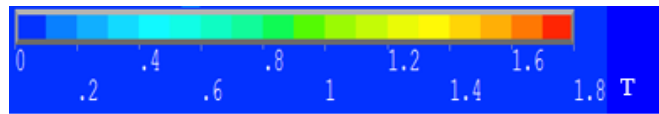

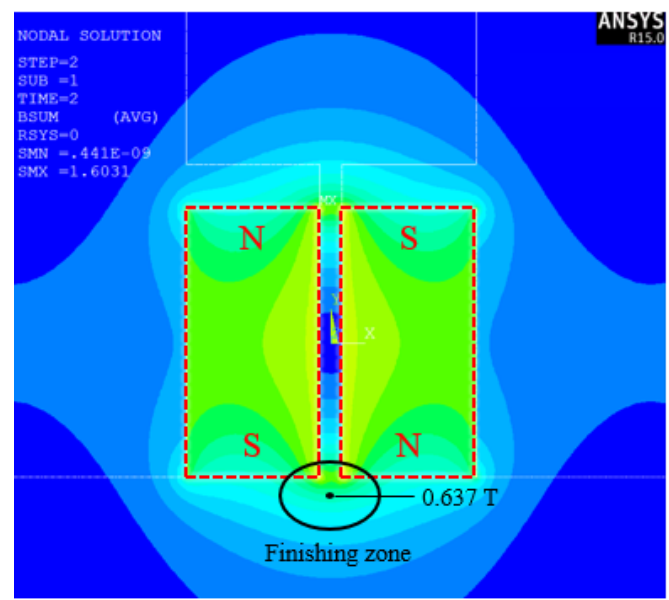

(a)

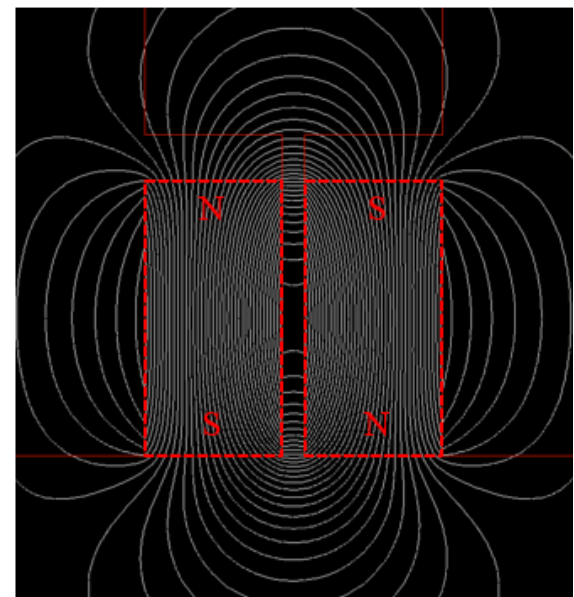

(b)

Figure 7: Magnetic field distribution analysis of the double-magnet apparatus showing the (a) magnetic flux distribution and (b) magnetic flux lines.

MFAF processes are typically used for non-magnetic workpiece. The workpiece used for experiments are stainless steel (SUS316) cuboids ( $25 \mathrm{~mm} \times 25 \mathrm{~mm} \times 10 \mathrm{~mm}$ ). Surface roughness reduction from an initial value of $0.5 \mu \mathrm{m} R a$ to below $0.05 \mu \mathrm{m} R$ a for both stainless steel and titanium alloy has been documented previously [4] and is therefore not of primary consideration within this work. Instead, the focus is on the material removal rate. Material removal rate is measured from two-dimensional profiles obtained using a Taylor Hobson stylus profilometer (Form Talysurf 2). The profiles consist of both polished and unpolished zones. The height difference between the two constitutes the material removal depth. All values of material removal depth reported is the average of 3 line measurements positioned $1 \mathrm{~mm}$ apart. Additionally, material removal rate is considered to be constant over the short polishing duration. As a result, material removal depth and material removal rate are proportional and both are therefore applicable for the purpose of validating the trends given by the model. An example of the surface profile is shown in Figure 8.

To validate the material removal rate model, polishing experiments were conducted by varying the properties of the finishing media. The median size of carbonyl iron particle is kept constant at $8 \mu \mathrm{m}$, while the alumina abrasive size is varied between $0.6 \mu \mathrm{m}$ and 15 $\mu \mathrm{m}$ diameter. The range of abrasive size is selected such that it contains values that are 
within and beyond the maximum allowable abrasive size. Secondly, the abrasive volume was varied using finishing media with abrasive-to-carbonyl-iron volumetric ratio, $V_{\mathrm{abr}} / V_{\mathrm{Cl}}$, ranging from 0 to 0.768 . Note that 0.259 corresponds to the abrasive saturation as given by the abrasive volume criterion in Equation 3. The experimental conditions are shown in Table 1 and the relationship between abrasive mass and $V_{\mathrm{abr}} / V_{\mathrm{Cl}}$ are shown in Table 2. Volumetric ratio in Table 2 is calculated based on a density of $7.87 \mathrm{~g} / \mathrm{cm}^{3}$ for iron and $4.1 \mathrm{~g} / \mathrm{cm}^{3}$ for alumina.
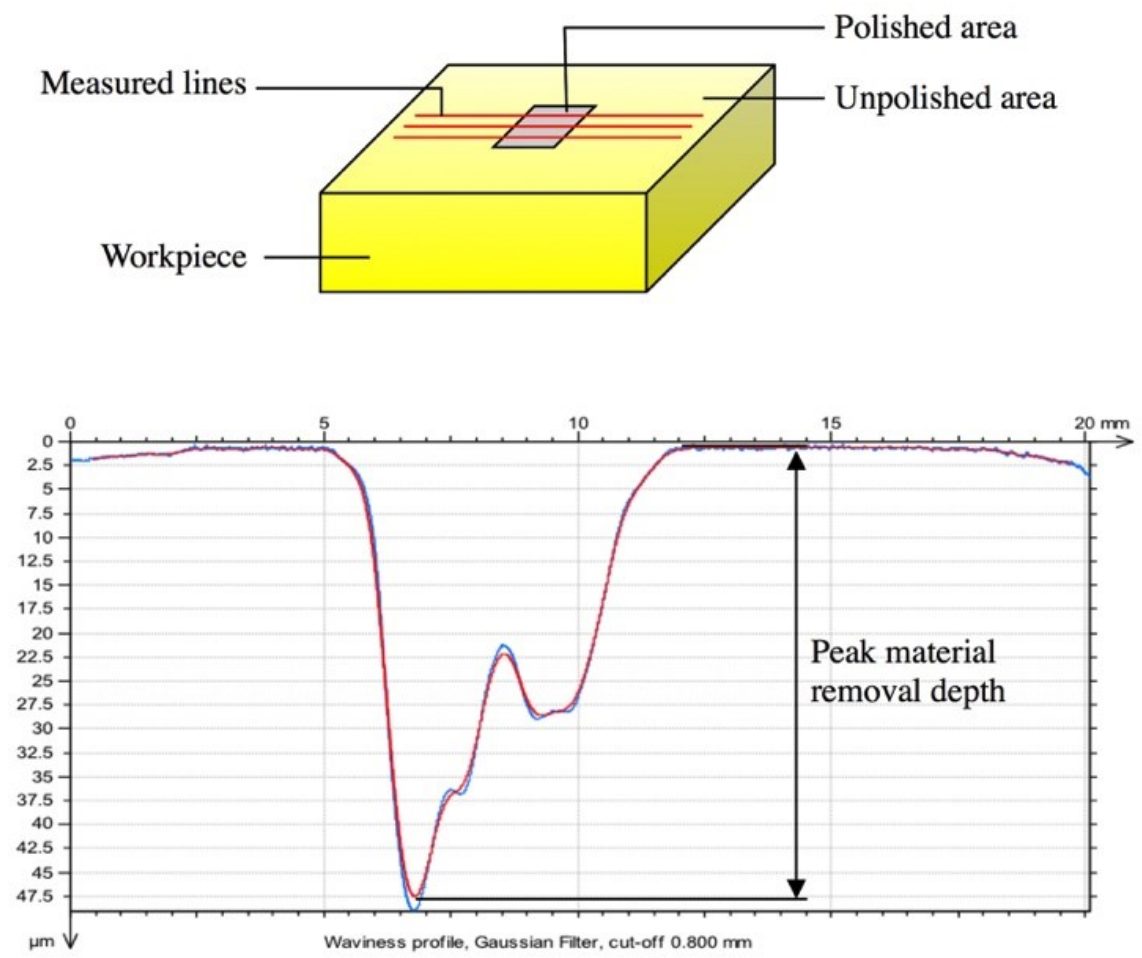

Figure 8: Surface profile measurement to determine material removal depth.

Table 1 Experimental conditions for double-magnet MFAF process.

\begin{tabular}{ll}
\hline Parameters & Values \\
\hline Polishing unit & \\
Magnet-to-magnet gap $(\mathrm{mm})$ & 1 \\
Magnet-to-workpiece gap $(\mathrm{mm})$ & 1 \\
\hline
\end{tabular}




\begin{tabular}{ll}
\hline Spindle revolution $\left(\mathrm{min}^{-1}\right)$ & 300 \\
Polishing time $(\mathrm{s})$ & 300 \\
\hline Finishing media & 20 \\
Carbonyl iron particles mass $(\mathrm{g})$ & $0-8.0$ \\
Alumina abrasives mass $(\mathrm{g})$ & 8 \\
Carbonyl iron particles median size $(\mu \mathrm{m})$ & $0.6-15$ \\
Alumina abrasives median size $(\mu \mathrm{m})$ & 8 \\
Water mass $(\mathrm{g})$ & \\
\hline
\end{tabular}

Table 2 Alumina abrasive mass selected and the corresponding $V_{\mathrm{abr}} / V_{\mathrm{cl}}$ values.

\begin{tabular}{cc}
\hline Alumina abrasive mass $(\mathbf{g})$ & Corresponding $V_{\mathrm{abr}} / V_{\mathrm{Cl}}$ \\
\hline 0 & 0 \\
0.3 & 0.029 \\
1.0 & 0.096 \\
2.0 & 0.192 \\
2.5 & 0.240 \\
2.7 & 0.259 \\
3.0 & 0.288 \\
4.0 & 0.384 \\
8.0 & 0.768 \\
\hline
\end{tabular}

\section{Comparison of theoretical and experimental results}

4.1 Calculation of constants 
A partial set of 5 data points were used to regressively calculate the four constants in the model, which are the removal factors $k_{\mathrm{Cl}}$ and $k_{\mathrm{abr}}$, and the force factors $f_{\mathrm{Cl}}$ and $f_{\mathrm{abr}}$. The force factor $f_{\mathrm{CI}}$ was arbitrarily set to 1 while other values were obtained iteratively based on the conditions $k_{\mathrm{Cl}} \gg k_{\mathrm{abr}}$ and $f_{\mathrm{Cl}} \gg f_{\mathrm{abr}}$. Finally, the values of $k_{\mathrm{Cl}}$ and $k_{\mathrm{abr}}$ were scaled such that the magnitude of values given by the model matched the magnitudes of experimental results. Table 3 shows the selected values of the four constants. The values of the four constants were then used to generate theoretical curves to fit against the remaining data points for validation.

Table 3 Values selected for the four constants in the model.

\begin{tabular}{lll}
\hline Constants & Values & Units \\
\hline
\end{tabular}

Removal factors

$\begin{array}{lll}k_{\mathrm{Cl}} & 1.5 \times 10^{4} & \text { Dimensionless } \\ k_{\mathrm{abr}} & 1.5 \times 10^{6} & \text { Dimensionless }\end{array}$

Force factors

$\begin{array}{ccc}f_{\mathrm{Cl}} & 1 & \mathrm{NT}^{-2} \mathrm{~m}^{-6} \\ f_{\mathrm{abr}} & 1 \times 10^{-8} & \mathrm{NT}^{-1} \mathrm{~m}^{-3}\end{array}$

\subsection{Abrasive size}

Figure 9 shows the graph of material removal depth against alumina abrasive size, which includes the experimental data points and the theoretical curves generated by the model based on the values of constants shown in Table 3. There are two sets of data, which correspond to two different $V_{\mathrm{abr}} / V_{\mathrm{Cl}}$ values. The theoretical curves consist of the base model and the extension for abrasive size criterion. The vertical dotted line at $D_{\mathrm{abr}}=3.2$ $\mu \mathrm{m}$ indicates the maximum allowable abrasive size according to Equation 2, which therefore also serve as the transition between the base model and the extension. According to the theoretical curves, the material removal rate decreases with an increasing abrasive size, which is supported by the trend exhibited by the experimental results. In addition, the theoretical curves show that the material removal rate is lower when the abrasive-to-carbonyl-iron volumetric ratio, $V_{\mathrm{abr}} / V_{\mathrm{Cl}}$ is reduced, which is also consistent with the experimental results. 


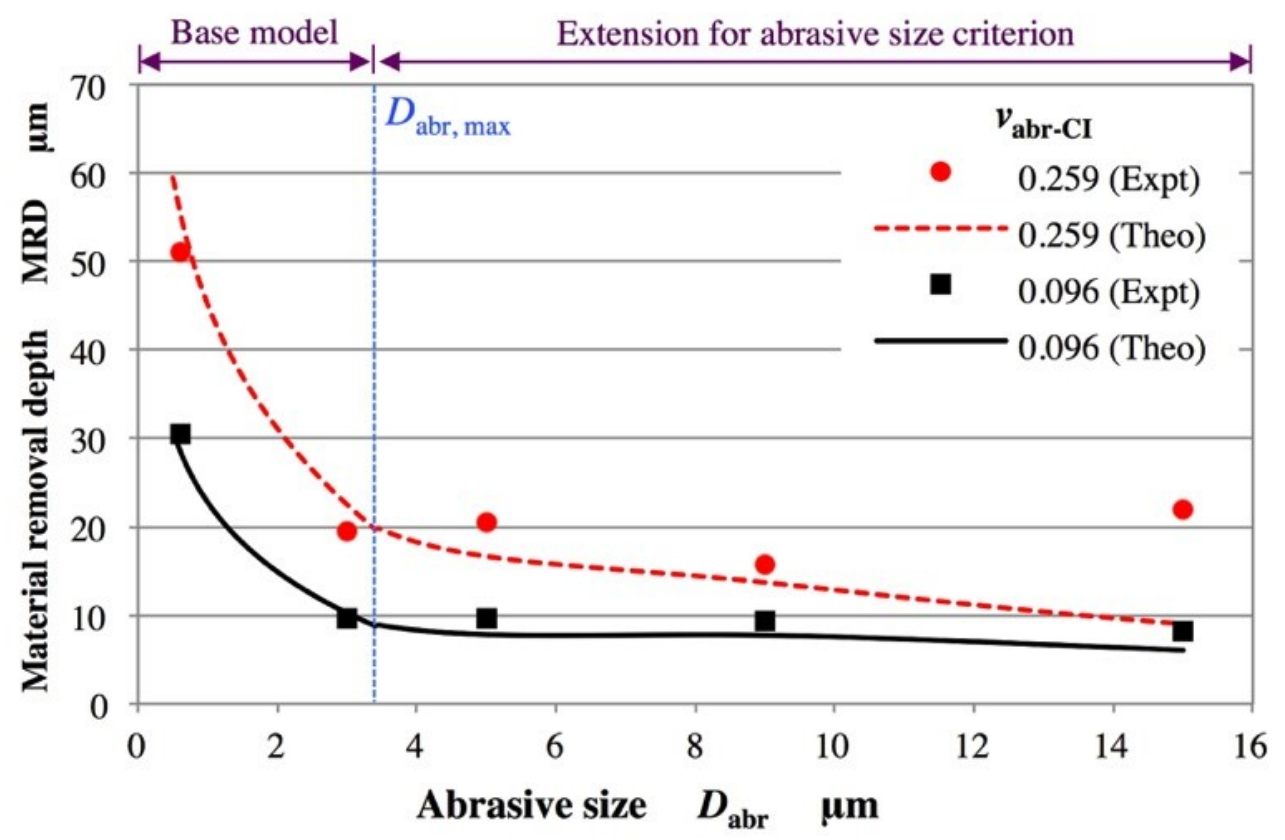

Figure 9: Experimental results and theoretical curves of material removal depth against abrasive size.

\subsection{Abrasive concentration}

Figure 10 shows the graph of material removal depth against alumina abrasive concentration, which is represented by the abrasives-to-carbonyl-iron volumetric ratio, $V_{\mathrm{abr}} / V_{\mathrm{Cl}}$. Similarly, the graph consists of experimental data points and theoretical curves for two different abrasive sizes. The theoretical curves consist of the base model and the extension for abrasive volume criterion. The vertical dotted line at $V_{\text {abr }} / V_{\mathrm{Cl}}=0.259$ indicates the abrasive saturation as given by Equation 3, which is therefore also the transition between the base model and the extension. According to the model, material removal rate initially increases with an increasing $V_{\text {abr }} / V_{\mathrm{Cl}}$ up to the point of abrasive saturation at 0.259 . The theoretical model suggests that further increment of $V_{a b r} / V_{\mathrm{Cl}}$ beyond this value will result in a reduction of material removal rate. Thus, the model suggests that a maximum material removal rate exists at the point of abrasive saturation. The same trend is suggested for both abrasive sizes, though the material removal rate is higher when the abrasive size is smaller, consistent with trend seen in the previous graph. These are in good agreement with the experimental results, where a maximum material removal rate was observed near the theoretical maximum at $V_{\mathrm{abr}} / V_{\mathrm{Cl}}=0.259$. Similar observations have been reported in another MFAF process [22]. In practice, the 
point of maximum material removal rate can be expected to fall within a range of values rather than at a single point, due to the stochastic nature of randomly distributed abrasive size.

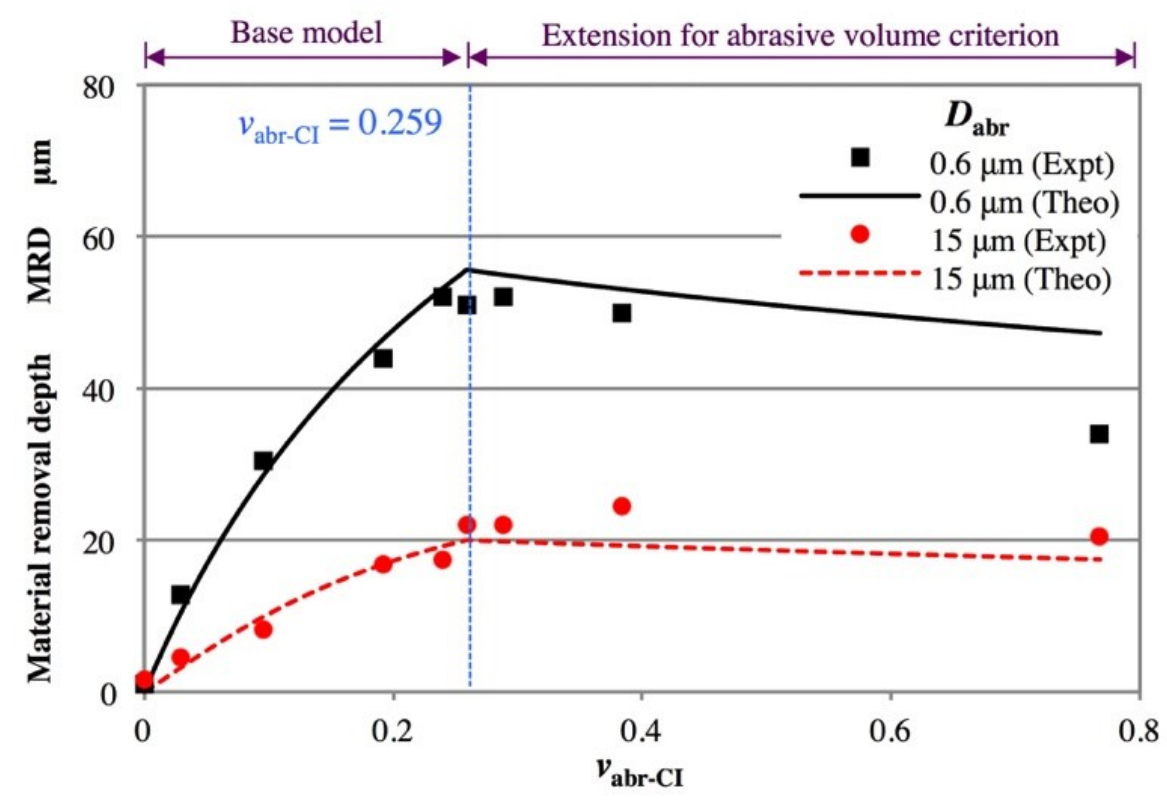

Figure 10: Experimental results and theoretical curves of material removal depth against abrasive-to-carbonyl-iron volumetric ratio, $\mathrm{Vabr} / \mathrm{VCl}$.

\section{Discussion}

The model is agnostic to workpiece geometry, as long as there is no feature that prevents media flow into the finishing zone. One such feature will be a protrusion on the flat surface. Surface morphology may affect the model, depending on how different it is compared to the conditions tested in this work. For example, a very rough surface of an electron beam melting workpiece may see material removal preferentially at the valleys, which creates deeper valleys instead of polishing the surface uniformly. Under those conditions, the proposed model may not be valid.

\section{Conclusions}

This paper proposed and developed a material removal rate model for magnetic fieldassisted finishing based on media properties. The conclusions and highlights are summarized below: 
- By modelling the number of active particles in the polishing media and interactions between them, a semi-empirical equation for material removal of a magnetic fieldassisted finishing process has been developed.

- Interactions between particles are modelled based on particle packing theory, assuming rigid spherical particles.

- The proposed model suggests that optimal material removal occurs when particles are in a close-packed condition, when abrasive-to-carbonyl-iron volumetric ratio is 0.259 .

- Trends of (1) material removal rate against abrasive size and (2) material removal rate against abrasive concentration as given by proposed model have been validated experimentally.

- A similar approach may be adopted for other variants of magnetic field-assisted finishing that utilizes media consisting of two different particle types.

There may be opportunities to improve the model by considering the following:

- More realistic, physics-based interactions between particles in the finishing media.

- Distributed particle size, instead of uniform size in current model.

The model can potentially reduce physical trials in developing a magnetic field-assisted finishing process for a new component, resulting in cost and time savings. Future work will be conducted on the concrete application of the model in aerospace industry.

\section{Acknowledgment}

The authors would like to acknowledge Agency of Science, Technology and Research $\left(A^{*} S T A R\right)$, Singapore for sponsoring the doctorate programme that enabled the completion of the work reported, and Singapore Institute of Manufacturing Technology (SIMTech) for supporting the work in its state-of-the-art research facility.

\section{References}

[1] Kordonski, W., \& Gorodkin, S. (2011). Material removal in magnetorheological finishing of optics. Applied Optics, 50(14), 1984-1994.

[2] Shinmura, T., Takazawa, K., Hatano, E., Matsunaga, M., \& Matsuo, T. (1990). Study on magnetic abrasive finishing. CIRP Annals - Manufacturing Technology, 39(1), 325-328.

[3] Sato, T., Kum, C. W., \& Ng, S. T. (2014). Micro-cracks removal on edge surface of thin glass sheet using magnetorheological finishing. Advanced Materials Research, 1017, 553-558. 
[4] Sato, T., Kum, C. W., \& Venkatesh, V. C. (2013). Rapid magneto-rheological finishing of Ti$6 \mathrm{Al}-4 \mathrm{~V}$ for aerospace components. International Journal of Nanomanufacturing, 9(5/6), 431445.

[5] Guo, J., Liu, K., Wang, Z., \& Tnay, G. L. (2017). Magnetic field-assisted finishing of a mold insert with curved microstructures for injection molding of microfluidic chips. Tribology International, 114, 306-314.

[6] Guo J. , Tan Z. E. E., Au K. H., \& Liu, K. (2017). Experimental investigation into the effect of abrasive and force conditions in magnetic field-assisted finishing. The International Journal of Advanced Manufacturing Technology, 90(5-8), 1881-1888.

[7] Yamaguchi, H., \& Shinmura, T. (1999). Study of the surface modification resulting from an internal magnetic abrasive finishing process. Wear, 225, 246-255.

[8] Yamaguchi, H., Riveros, R. E., Mitsuishi, I., Takagi, U., Ezoe, Y., Yamasaki, N., \& Hashimoto, F. (2010). Magnetic field-assisted finishing for micropore $X$-ray focusing mirrors fabricated by deep reactive ion etching. CIRP Annals - Manufacturing Technology, 59(1), 351-354.

[9] Singh, A. K., Jha, S., \& Pandey, P. M. (2011). Design and development of nanofinishing process for 3D surfaces using ball end MR finishing tool. International Journal of Machine Tools and Manufacture, 51(2), 142-151.

[10] Brinksmeier, E., Riemer, O., \& Gessenharter, A. (2006). Finishing of structured surfaces by abrasive polishing. Precision Engineering, 30(3), 325-336.

[11] Evans, C. J., Paul, E., Dornfeld, D., Lucca, D. A., Byrne, G., Tricard, M., \& Mullany, B. A. (2003). Material removal mechanisms in lapping and polishing. CIRP Annals - Manufacturing Technology, 52(2), 611-633.

[12] Preston, F. W. (1927). The theory and design of plate glass polishing machines. J. Soc. Glass Tech., 11, 214.

[13] Buijs, M., \& Korpel-van Houten, K. (1993). A model for lapping of glass. Journal of Materials Science, 28(11), 3014-3020.

[14] DeGroote, J. E., Marino, A. E., Wilson, J. P., Bishop, A. L., Lambropoulos, J. C., \& Jacobs, S. D. (2007). Removal rate model for magnetorheological finishing of glass. Applied Optics, 46(32), 7927-7941.

[15] Misra, A., Pandey, P. \& Dixit, U. S. (2017). Modeling of material removal in ultrasonic assisted magnetic abrasive finishing process. International Journal of Mechanical Sciences, 131-132, 853-867.

[16] Misra, A., Pandey, P. \& Dixit, U. S. (2017). Modeling of surface roughness in ultrasonic assisted magnetic abrasive finishing process. International Journal of Mechanical Sciences, 133, 344-356. 
[17] Luo, J., \& Dornfeld, D. (2001). Material removal mechanism in chemical mechanical polishing: theory and modeling. Semiconductor Manufacturing, IEEE Transactions, 14(2), 112-133.

[18] Jeng, Y. R., \& Huang, P. Y. (2005). A material material removal rate model considering interfacial micro-contact wear behavior for chemical mechanical polishing. Journal of Tribology, 127(1), 190-197.

[19] Kanish, T. C., Narayanan, S., Kuppan, P. \& Denis Ashok, S. (2017). Investigations on the finishing forces in magnetic field assisted abrasive finishing of SS316L. Procedia Engineering, 174, 611-620.

[20] Ellis, A. B., Geselbracht, M. J. \& Johnson, B. J. (1995). Teaching general chemistry - a materials science companion. Washington, DC. American Chemical Society.

[21] Brown, N. J., Baker, P. C., \& Maney, R. T. (1982). Optical polishing of metals. In 25th Annual Technical Symposium, International Society for Optics and Photonics, 42-57.

[22] Sidpara, A., \& Jain, V. K. (2012). Theoretical analysis of forces in magnetorheological fluid based finishing process. International Journal of Mechanical Sciences, 56(1), 50-59. 